\title{
Estrategias de enseñanza y tecnologías en publicaciones previas a la forzosa virtualización de la educación
}

\author{
Majul, Sofía*; De Seta, Damián*; Borgobello, Ana*
}

\section{Resumen}

Se presenta un análisis de la relación TIC-estrategias de enseñanza en escritos previos a la pandemia de covid-19. La muestra de 61 artículos se organizó en dos conjuntos de datos; por un lado, se agruparon los que hablaban de las claves de búsqueda de forma general, y por otro, se agruparon aquellos que las abordaban en particular, es decir, especificaban una TIC y una estrategia. los resultados destacan la necesidad de reforzar las estrategias de enseñanza con integración de TIC ya que se sostiene que su uso favorece el aprendizaje. A partir de la revisión bibliográfica se concluye que la incorporación de TIC en educación conlleva un trabajo de antelación que se habría visto ampliamente dificultado al inicio de la pandemia por la inmediatez del trabajo docente debido a la virtualización forzosa de la educación.

Palabras clave: estrategias de enseñanza; TIC; modelo pedagógico; modalidades físico-virtuales

Este artículo se enmarca, por un lado, en una línea de investigación radicada en IRICE-CONICET/UNR «Interacción sociocognitiva en entornos virtuales» (2020-2021) y, por el otro, en el Proyecto «Prácticas educativas con TIC» (2020-2023, Res.CS125/21), radicado en la Facultad de Psicología de la UNR ambos dirigidos por la Dra. Ana Borgobello. Recibido el 22/03/2021, aprobado el 01/09/2021 y publicado el 19/10/2021.

DOI: https://doi.org/ 10.33255/3263/1025

Autoría: *Instituto Rosario de Investigaciones en Ciencias de la Educación (CONICET/UNR), Argentina. Contacto: borgobello@irice-conicet.gov.ar 
Teaching strategies and technologies in papers published previously to the virtualization of education during pandemic time

\begin{abstract}
Conceptual relationship between ICT and teaching strategies determine learning construction. This paper aims to analyze papers about this topic. From the 61 articles sample, it was organized into two data sets: papers with search key concepts in title, abstract and keywords, and particular ICT and particular strategies described. The results highlight the need to reinforce teaching strategies with integration of ICT since it is argued that it promotes learning construction. From the bibliographic review it is concluded that ICT incorporation in education implies previous pedagogical planning work. This previous work would have not been done at least in the beginning of pandemic time.
\end{abstract}

Keywords: teaching strategies; ICT; pedagogical model; physic-virtual modalities

\title{
Estratégias de ensino e tecnologias em publicações anteriores à virtualização forçada da educação
}

\section{Resumo}

Foi realizada uma análise da relação entre as TIC e as estratégias de ensino em escritos anteriores a pandemia de Covid-19, visto que é considerada de suma relevância para propiciar a construção da aprendizagem. A amostra de 61 artigos foi organizada em dois conjuntos de dados, de um lado, foram agrupados aqueles que falavam das chaves de busca de forma geral e, de outro, agruparam-se aqueles que as tratavam em particular, ou seja, especificavam uma TIC e uma estratégia. Os resultados evidenciam a necessidade de reforçar as estratégias de ensino com a integração das TIC, uma vez que se argumenta que seu uso favorece a aprendizagem. A partir da revisão bibliográfica conclui-se que a incorporação das TIC em educação implica um trabalho antecipado que teria sido muito dificultado no início da pandemia pelo imediatismo do trabalho docente devido à virtualização forçada da educação.

Palavras-chave: Estratégias de ensino, TIC, modelo pedagógico, modalidades físicovirtuais 


\section{Introducción}

La modalidad virtual de enseñanza se caracteriza por potencialidades como la flexibilidad de tiempos, espacios y medios de comunicación con estudiantes que, generalmente, se encuentran dispersos geográficamente, siendo altamente sistematizada y destacándose por la anticipación y planificación de materiales y actividades. Durante el período de aislamiento a raíz de la pandemia de COVID-19, las características de esta modalidad fueron ampliamente aprovechadas, pero sin la planificación y anticipación propios de la modalidad, justamente debido a la circunstancia de crisis dada. Este formato caracterizado por la «improvisación» y la organización institucional en emergencia llevaron, incluso, a hablar irónicamente de la educación a distancia en contraposición al coronateaching o enseñanza remota de emergencia (ERE) (Ramos, 2020; Pérez, 2020).

A partir de la ERE, la sociedad se encontró frente a una compleja situación en relación con las estrategias de enseñanza y el uso de TIC (tecnologías de la información y la comunicación) en los distintos niveles educativos. Si partimos de la mirada prospectiva que la educación asume frente a un futuro todavía no determinado, podemos decir que, desde los tiempos de pandemia y aislamiento social del 2020 , ese futuro indeterminado se ha adelantado, de forma inédita, convirtiéndose en un presente (De Luca, 2020), en el que el uso de TIC se ha visto intensificado. Sin embargo, como plantean Manso et al. (2019), a pesar de que existe consenso en pensar que habitamos una sociedad centrada en las TIC y caracterizada por nuevos modos de administración de la información, esta misma sociedad parece reclamar estrategias de enseñanza y aprendizaje renovadas, encontrando dificultades para modificar los tradicionales estilos docentes de tipo transmisivo.

En tal sentido, a nivel institucional, Copertari (2020) sostiene que existe demanda previa a la pandemia de implementación de diseños curriculares flexibles en términos de tiempos y espacios, innovadores, democráticos, con acciones pedagógicas y didácticas que consideren otras formas de presencialidades y con uso de entornos educativos no tradicionales. En función de ello, más allá de la crisis, es posible plantear que, incluso antes de la virtualización forzosa, se reclamaban espacios colectivos de reflexión respecto de las estrategias de enseñanza y su relación con las TIC (Borgobello et al., 2019).

Los espacios de reflexión requerirían compartir experiencias en contextos académicos así como literatura especializada. Dada la multiplicidad y heterogeneidad de literatura científica sobre estrategias de enseñanza y TIC, se hace necesario analizar distintas definiciones en uso. A partir del análisis 
realizado para escribir este texto, podemos decir que tan solo con el primer concepto, estrategias de enseñanza, se encuentran al menos tres aspectos que complejizan su abordaje y dificultan la posibilidad de obtener claridad y precisión: existen diversos modos de nombrarlo; se lo menciona sin ser definido, $y$, cuando se define, subyacen perspectivas teóricas diversas.

Como se dijo, estrategias de enseñanza, así como las diversas formas de mencionarlas, es un concepto que suele darse por sabido sin definirse (e.g. Carranza y Caldera 2018; Jenaro et al., 2018) o se define escasamente en los textos. Para ilustrar esta diversidad, se menciona haciendo referencia a un objeto que parece ser común: estrategias de enseñanza (Anijovich y Mora, 2010; Pérez y La Cruz, 2014); estrategias docentes (Ávila et al., 2010); estrategias pedagógicas (Miranda, 2015; Turizo, 2011; Lucumi et al., 2015); estrategias didácticas (Asinsten, 2013; Concari, 2014; Delgado y Solano, 2009; Nossa, 2007; Ramón y Vilchez, 2019); estrategias educativas (Casado, 2018; Lizcano et al., 2019; Martínez-Palmera et al., 2018; Sánchez-Vera et al., 2019; Ventura, 2013); estrategias de enseñanza-aprendizaje (Cabrera et al., 2016; Saza et al., 2019); y estrategias de enseñanza y aprendizaje (Estéfano, 2018; López et al., 2018; Martín y Tourón, 2017). Si bien estas denominaciones pueden ser producto de énfasis distintos a nivel teórico, suelen ser tomadas como sinónimos; incluso en un mismo texto se las nombra de distintas maneras, sin diferenciarlas (e.g. Martín y Tourón, 2017).

Cuando se define conceptualmente estrategias de enseñanza, se lo hace de forma heterogénea, aludiéndose a distintos tipos de dimensiones teóricas. Por ejemplo, a veces engloban un conjunto de recursos (Turizo, 2011); otras se basan en conceptos específicos como aprendizaje colaborativo (Miranda, 2015); en ocasiones se usa la palabra estrategia como sinónimo de técnicas o recursos (Delgado y Solano, 2009) o como conjunto de procedimientos (Rivero et al., 2013); asimismo, se relacionan con aspectos institucionales vinculados a la innovación (Marín et al., 2017; Martínez-Usarralde et al., 2018).

Frente a la multiplicidad de términos y perspectivas, tomamos como criterio de síntesis la posición teórica planteada por Anijovich y Mora (2010) y su denominación de estrategias de enseñanza. Esta decisión responde a que las autoras se oponen a la idea de estrategia como mera técnica enfocándose en el concepto enmarcado en posicionamientos teóricos y en contextos en los que las mismas tienen lugar, aun cuando quienes utilizan este concepto puedan no ser plenamente conscientes de los fundamentos teóricos y contextuales que subyacen. Las autoras entienden las estrategias como modos de pensar las clases, opciones y posibilidades para que algo sea enseñado, decisiones creativas o una variedad de herramientas artesanales con las que contamos 
para preparar una clase. Advierten que si bien el concepto suele aparecer en la bibliografía asociado a la didáctica, existen interpretaciones ambiguas. Sintéticamente, definen a las estrategias de enseñanza como el conjunto de decisiones que se toman desde un rol docente para orientar la enseñanza con el fin de promover el aprendizaje de sus estudiantes. Se trata de orientaciones generales acerca de cómo enseñar un contenido disciplinar considerando qué quieren que comprendan, por qué y para qué quieren que lo hagan.

Para las autoras, en tanto decisiones, las estrategias de enseñanza engloban dos dimensiones: por un lado, una dimensión de reflexión, y por el otro, una dimensión de acción. Según sea la particularidad de cada caso, será predominante una dimensión sobre la otra, dependiendo de cómo se defina el concepto; esto es, subordinándose a la influencia que tenga el contexto tanto institucional como discursivo en el que se tomen las decisiones. Esta denominación y definición permiten, a nuestro parecer, resolver las dificultades con respecto a la claridad y precisión conceptuales y las dificultades que surgen frente a la heterogeneidad de definiciones existentes, brindando la articulación de las dimensiones en juego.

En cuanto a la literatura que aborda la temática estrategias de enseñanza específicamente en relación con las TIC, este panorama teórico se complejiza aún más. Escandell et al. (2004) sostienen que el uso de TIC no refiere a la incorporación de ordenadores $u$ otras tecnologías en el proceso educativo, sino que más bien implica una etapa avanzada, donde el énfasis se pone en la conexión a internet. Desde esta perspectiva, afirman que las TIC adquieren diversos matices, entre los que se encuentra la posibilidad de lenguajes expresivos que permiten nuevas representaciones, entre las que se puede incluir los multimedia e hipertextos, y su posibilidad de interconexión. A diferencia de la web 1.0 que, como apunta García Aretio (2007), se trataba más de lectura y con páginas bastante estáticas, la web 2.0 implicó no solo lectura sino también escritura, con páginas dinámicas de contenidos abiertos. En este sentido, Domínguez y Llorente (2009) plantean que la web 2.0 proporcionó espacios de interacción social posibilitando la participación colectiva y gratuita mediante tecnologías abiertas, flexibles y fáciles de utilizar.

A pesar de estos cambios, el uso de TIC no se asocia directamente a un modelo pedagógico determinado. Las TIC están vinculadas a la socialización de información a través de la comunicación y no fueron diseñadas específicamente para dar lugar a procesos educativos. Enfatizando las problemáticas de las dinámicas de inserción, sustentabilidad económica y social, autoras como Guarnieri (2018) plantean que se puede pensar en las TIS (tecnologías para la inclusión social), en las que el desarrollo e implementación de tecnologías 
incluye productos, procesos y organización. Desde posturas críticas se distingue a las TIC de conceptos como TAC (tecnologías para el aprendizaje y el conocimiento) y TEP (tecnologías para el empoderamiento y la participación) para mostrar la necesidad de abordar la especificidad de las tecnologías en los ámbitos educativos y de la generación de conciencia ciudadana, social, democrática y empoderada (Cabero et al., 2019).

A pesar de esta distinción conceptual, suele hablarse de TIC como herramientas para enseñar desde modelos pedagógicos tanto transmisivos como constructivistas. Para Ortiz (2013), el modelo pedagógico no ha sido definido con claridad en la literatura específica. Desde su definición, el mismo tiene diversos rasgos como la anticipación al trabajo pedagógico, y se concreta en distintos niveles, pero, fundamentalmente en el aula, respondiendo a necesidades históricas concretas. Es decir, se trata de un conjunto de representaciones ideales del mundo real de lo educativo. El concepto de modelo pedagógico tiene carácter teórico y fue creado para concebir idealmente los procesos de enseñanza y aprendizaje; por tanto, sería algo así como un paradigma que permite analizar, interpretar, orientar y transformar la educación. Díez (2012) sostiene que las TIC pueden ser utilizadas por el profesorado desde modelos innovadores, permitiendo responder a desafíos que propone la sociedad actual o manteniendo modelos pedagógicos tradicionales basados en la idea de adquisición de conocimiento y escasa interactividad.

Más allá del modelo pedagógico, al estudiar las estrategias de enseñanza en el marco de las TIC, parece haber consenso en entender que las modalidades aúnan un conjunto de estrategias ya sean mediadas o dadas en encuentros tradicionales en persona. Como polos prototípicos, aparecen la modalidad virtual y la presencial, encontrándose en medio la más diversa gama de combinaciones en tiempos y espacios que son denominadas en la literatura como bimodalidad (e.g. Villar, 2016), modelo híbrido (e.g. Koohang, 2004), blended learning (e.g. Carranza y Caldera, 2018), mixto (e.g. García y Ramírez, 2018) y semipresencial (e.g. Copertari y Sgreccia, 2011). Carranza y Caldera (2018) entienden que la bimodalidad o blended learning se caracteriza por aplicar un conjunto de estrategias de enseñanza provenientes de ambas tradiciones. Si bien se reconoce a la bimodalidad como la combinación de encuentros presenciales y virtuales (e.g. Villar, 2016), suele definirse en términos de la suma de los espacios o escenarios y tiempos, aunque no en sentido pedagógico propiamente dicho. Por esa razón, al igual que sucede con las estrategias, es difícil encontrar definiciones de modalidades de enseñanza más que a nivel operativo. En este sentido, Duch y Núñez (2016) sostienen que las estrategias docentes elegidas dependen de la centralidad de cada componente de la 
modalidad de la que se trate (virtual o presencial/en persona) en consonancia con el modelo pedagógico asumido o subyacente.

Guarnieri (2018) enfatiza la continuidad entre componentes virtuales y presenciales dando lugar a la idea de interactividad físico-virtual, entendiéndola como el vínculo intersubjetivo responsable mediatizado por las TIC que conforma una red socio-técnica generadora de multidireccionalidad de mensajes y objetos que se intercambian en marcos de trabajo preferentemente colaborativos, abiertos, democráticos y plurales. Destaca que para generar intercambio dialógico y habilitar la dimensión participativa de la democracia en espacios mediados por TIC, se hace necesario un diseño interactivo apropiado.

El diseño con el que se encuentran quienes participan en estos espacios se constituye a través de propuestas que integran, por un lado, aspectos pedagógicos o instruccionales, y por el otro, tecnológicos, dando lugar a lo que Coll et al. (2007) denominan diseño tecnopedagógico o tecnoinstruccional. Los elementos que componen estos diseños son los contenidos, los objetivos y las actividades de enseñanza y aprendizaje, como así también las consignas de trabajo plasmadas en orientaciones y sugerencias sobre el modo de realizarlas y las herramientas tecnológicas seleccionadas junto con sugerencias y orientaciones sobre cómo utilizarlas en el desarrollo de las actividades. Para estos autores, las TIC pueden entenderse como instrumentos de tipo psicológicos en sentido vigotskiano y no solo como tecnologías que se usan en sentido de exterioridad. A su vez, las TIC conllevan tanto posibilidades como limitaciones en la organización de la actividad conjunta académica, variando en relación con el equipamiento y los recursos tecnológicos que se dispongan. El uso de TIC se encuentra condicionado por normas, procedimientos y recomendaciones dadas en cada contexto institucional específico. Asimismo, la actividad conjunta puede pensarse como consecuencia de negociación y construcción de significados que se basan en expectativas y motivaciones, y no como meras reproducciones en un curso dado de los marcos institucionales de uso de TIC.

A partir del conjunto de conceptos hasta aquí trabajados, entendemos que se hace necesario reflexionar sobre la relación entre uso de TIC y estrategias de enseñanza, ya que la misma direcciona las actividades docentes que propician la construcción de aprendizajes. Desde esta perspectiva, nos preguntamos, sin pretensión de exhaustividad, acerca de las relaciones que pueden establecerse entre ambos conceptos en uso en la literatura científica especializada. 


\section{Materiales y métodos}

La revisión de la literatura se llevó a cabo en distintas fases. En primer lugar, realizamos una búsqueda en la base de datos Scopus utilizando la siguiente combinación de términos considerados claves de búsqueda: estrategias y TIC. En el archivo .pdf obtenido de la base de datos quedó consignado esto del siguiente modo: «estrategias» AND TIC AND (LIMIT-TO SRCTYPE,»j»). Al seleccionar los filtros consideramos solo artículos de acceso abierto en español y año de publicación (2017-2019), habiéndose hecho la búsqueda en octubre de 2019. Dado que la temática del uso de TIC se intensificó en las publicaciones debido a la situación de enseñanza remota de emergencia, decidimos mantener la búsqueda para poder trazar una suerte de línea de demarcación histórica que describa un antes y un después de la pandemia en la temática en textos científicos. La base de datos generó un archivo con los resultados de la búsqueda: 142 artículos en los que aparecían tanto TIC como estrategias en alguna parte del texto.

En la segunda fase procedimos al análisis del título, resumen y palabras clave para generar criterios de inclusión/exclusión, dejando en la base de datos definitiva 61 artículos sobre educación que mencionaban las claves de búsqueda o alguna TIC o estrategia de enseñanza en particular.

En la tercera fase de trabajo, Análisis descriptivo preliminar, cada uno de los artículos fue analizado en función de las claves de búsqueda y de una serie de variables construidas a partir de ese análisis preliminar de los datos. Se consignó si «TIC» o «tecnologías de la información y de la comunicación» y «estrategias» se encontraban en el título, resumen o palabras clave. Por otro lado, se identificó si el artículo versaba sobre una TIC y una estrategia de enseñanza en particular, y algunas características metodológicas de los estudios presentados: específicamente, si contenían resultados empíricos o se trataba de reflexiones teóricas y sugerencias generales o de artículos de revisión. Se analizó la frecuencia de aparición de cada categoría mencionada por separado.

En la cuarta fase de trabajo, realizamos un Análisis de los artículos destacados a partir de los cuatro que mencionaran por lo menos una vez estrategias y TIC en las variables título, resumen y palabras clave. Consideramos estos textos debido a que los términos clave de búsqueda se encuentran en los sectores más destacados de un artículo, mostrando así la especificidad respecto de la temática. En el análisis cualitativo de las características de los textos, se consignaron países en los que fueron realizados los estudios, nivel educativo al que se hacía mención y la relación que establecían entre la idea de estrategias y TIC.

Como quinta y última fase de trabajo con los datos, seleccionamos los artículos que mencionaran TIC y estrategias particulares considerándolos un 
segundo núcleo de análisis al que denominamos Análisis de particularidades de la relación TIC-estrategias de enseñanza. A partir de los doce artículos con estas características, se analizaron las relaciones establecidas entre la TIC y la estrategia de enseñanza encontradas en cada artículo. Desde la definición de Anijovich y Mora (2009) de estrategias de enseñanza, se describieron algunas de las decisiones y acciones destinadas a enseñar un determinado contenido a una persona o grupo. Específicamente nos centramos en qué se enseñaba y cómo se utilizaban las TIC en el contexto en el que se presentaban las mismas.

\section{Resultados}

\section{Análisis descriptivo preliminar}

En la primera parte de los resultados describimos un análisis preliminar de los datos en función de las variables construidas (véase Tabla 1). TIC fue mencionado con mayor frecuencia en palabras clave $(n=25,41 \%)$, seguido en resumen $(n=23,38 \%)$ y más escasamente en título $(n=14,23 \%)$.

Tabla 1. Descripción general de los artículos de la muestra

\begin{tabular}{l|c|c}
\multicolumn{1}{c|}{ Variable } & Frecuencia & $\%$ \\
\hline TIC en resumen & 23 & 38 \\
\hline TIC en título & 14 & 23 \\
\hline TIC en palabras clave & 25 & 41 \\
\hline TIC en particular & 19 & 31 \\
\hline Estrategias en título & 5 & 8 \\
\hline Estrategias en resumen & 8 & 13 \\
\hline Estrategias en palabras clave & 2 & 3 \\
\hline Estrategia particular & 32 & 52 \\
\hline Resultados empíricos & 50 & 82 \\
\hline Artículos de revisión & 11 & 18 \\
\hline
\end{tabular}

La aparición de la palabra estrategias es escasa en los apartados destacados de los artículos: en palabras clave aparece solo dos veces (3\%), en títulos cinco $(8 \%)$ y en resumen ocho $(13 \%)$. Esto puede deberse a que se mencionara cada estrategia en particular $(n=32,53 \%)$ en lugar de mencionarse el término. 
Adicionalmente, encontramos que cinco de los artículos (8\% de la muestra) que no mencionan resultados empíricos tampoco hablan de una TIC ni de alguna estrategia en particular (Ibáñez et al., 2018; Matesanz del Barrio y Ferreira, 2017; Miranda et al., 2018; Valverde, 2018; Valverde et al., 2018).

Solo cuatro de los artículos mencionan TIC y estrategias, es decir, ambas claves de búsqueda, en los lugares más visibles: títulos, resúmenes y/o palabras clave (Carranza y Caldera, 2018; Lizcano et al., 2019; Marín et al., 2017; Sánchez Otero et al., 2019). Solo uno de esos artículos menciona ambas claves de búsqueda en las seis variables construidas (Sánchez Otero et al., 2019). El análisis de este grupo de artículos se profundiza en un apartado debajo.

Solo doce artículos de la muestra (20\%) hablan simultáneamente de una TIC específica y una estrategia de enseñanza en particular, por lo que también hemos decidido hacer un análisis pormenorizado de estos artículos, que se encuentra a continuación. A excepción de uno de estos artículos, una revisión bibliográfica basada en experiencias recogidas de publicaciones (Basso et al., 2018), todos los demás muestran resultados propios del trabajo de investigación o docencia.

En cuanto a los resultados empíricos desde el relato de experiencias educativas, los análisis cualitativos, cuantitativos o experimentales suman el $82 \%$ de la muestra $(n=50)$, es decir, solo un $18 \%$ de los artículos $(n=11)$ escriben únicamente desde posicionamientos teóricos o sugerencias generales sobre la temática abordada. Los artículos de revisión $(n=11,18 \%)$ encontrados en la muestra se dividen en dos grupos: seis de ellos $(10 \%)$ tienen resultados empíricos a partir de muestras constituidas por publicaciones y los cinco restantes $(8 \%)$ solamente se basan en posicionamientos teóricos o sugerencias generales.

\section{Análisis de los artículos destacados}

Analizamos a continuación algunas de las características del subconjunto de artículos que contienen en título, resumen y/o palabras clave ambos términos de la búsqueda en al menos uno de esos apartados. Podría considerarse que estos textos, debido a dónde se ubican los términos de búsqueda, abordan abiertamente la problemática de interés de este artículo.

Se destaca que la filiación institucional de los autores de tres de los artículos está radicada en Colombia (Lizcano et al., 2019; Marín et al., 2017; Sánchez-Otero et al., 2019) y el restante en México (Carranza y Caldera, 2018).

Dos artículos refieren a estudios sobre análisis institucionales del uso de TIC en educación superior (Carranza y Caldera, 2018; Marín et al., 2017) y los otros dos hacen revisiones de artículos. De los artículos de revisión, uno de 
ellos analiza cómo se fueron transformando las estrategias pedagógicas en la educación superior a partir de la inserción de las TIC (Sánchez Otero et al., 2019) y el otro se centra en la relación entre aprendizaje colaborativo y TIC (Lizcano et al., 2019).

Observamos que en los cuatro artículos se menciona que el uso de TIC favorece el aprendizaje. En cuanto a las TIC, se habla de ellas en forma general, sin establecer demasiadas especificaciones sobre aspectos tecnológicos o detalles de actividades en términos tecnopedagógicos. Refiriendo a las estrategias de enseñanza, las mismas se presentan en términos generales, aunque en dos de los artículos se las considera en relación con conceptos de aprendizaje asociados a teorías educativas reconocidas. Lizcano et al. (2019) abordan la generación de tareas vinculadas al aprendizaje colaborativo y Carranza y Caldera (2018) estudian la percepción de estudiantes de nivel universitario sobre sus procesos de aprendizaje significativo en relación con las estrategias de enseñanza que emplean sus docentes en el uso de aulas virtuales como complemento del aula tradicional de clases o blended learning.

Los cuatro artículos destacan, a su vez, la necesidad de reforzar las estrategias de enseñanza con integración de TIC. Sánchez Otero et al. (2019) consideran que las TIC incrementan las modalidades comunicativas favoreciendo entornos flexibles y eliminando barreras espaciales y temporales, aunque requieren alfabetización digital. Para Lizcano et al. (2019), la necesidad de reforzar las estrategias con TIC se debe a que favorecen la construcción social del aprendizaje facilitando la comunicación, el trabajo grupal y estilos activos propios del aprendizaje colaborativo. Carranza y Caldera (2018) afirman que la bimodalidad enfocada en el aprendizaje significativo, modo pertinentemente de utilizarla según los autores, es efectiva desde el punto de vista del estudiantado. Marín et al. (2017) consideran que las TIC en educación superior incrementan la capacidad de investigación, aumentando la promoción de la innovación, aunque la gestión de estas requiere formación del profesorado para que las prácticas pedagógicas lleven, por ejemplo, a responder en forma ágil y oportuna.

\section{Análisis de las particularidades de la relación tecnologías-estrategias de enseñanza}

Esta última parte de los resultados se centra en los doce artículos que mencionaban TIC y estrategias particulares considerándolos un núcleo de análisis específico. A continuación, se analizan las relaciones establecidas entre tecnologías y la estrategia de enseñanza mencionada en cada artículo desde las categorías vinculadas al concepto de estrategias de enseñanza de Anijovich y 
Mora (2010). Así, se destacan las decisiones y acciones destinadas a enseñar un determinado contenido (el qué), el rol de la TIC (desde el cómo) y otras características propias de cada publicación (Cuadro 1).

Cuadro 1. Descripción de los textos que relacionan tecnologías con estrategias de enseñanza

\begin{tabular}{|c|c|c|c|c|c|c|c|}
\hline $\begin{array}{l}\text { Primer } \\
\text { autor/a }\end{array}$ & País & Tecnologias & Estrategias: Cómo & Estrategias: Qué & $\begin{array}{c}\text { Grupal } \\
\text { /Individual }\end{array}$ & $\begin{array}{l}\text { Formación } \\
\text { docente }\end{array}$ & Tipo de escrito \\
\hline $\begin{array}{l}\text { Aarón et al. } \\
\text { (2018) }\end{array}$ & Colombia & Plataforma SMILE & $\begin{array}{l}\text { Tarea: construcción de preguntas y } \\
\text { respuestas }\end{array}$ & Disciplinar & 1 & No & $\begin{array}{l}\text { Informe con relato de } \\
\text { experiencia }\end{array}$ \\
\hline $\begin{array}{l}\text { Arancibia et al. } \\
\text { (2019) }\end{array}$ & Chile & $\begin{array}{l}\text { Pizarra digital } \\
\text { interactiva (PDI) }\end{array}$ & Trabajo en clases con PDI & Lectoescritura & G & No & $\begin{array}{l}\text { Informe de estudio } \\
\text { experimental }\end{array}$ \\
\hline $\begin{array}{l}\text { Jenaro et al. } \\
\text { (2018) }\end{array}$ & España & Moodle & Tareas: según tema & Disciplinar & 1 & No & $\begin{array}{l}\text { Informe de estudio } \\
\text { cuantitativo }\end{array}$ \\
\hline $\begin{array}{l}\text { Martínez et al. } \\
\text { (2018) }\end{array}$ & España & $\begin{array}{l}\text { Blog y } 2 \text { redes } \\
\text { sociales }\end{array}$ & $\begin{array}{l}\text { Tarea: mantener actualizadas redes } \\
\text { sociales }\end{array}$ & $\begin{array}{l}\text { Competencias digitales } \\
\text { para profesionales }\end{array}$ & 1 & No & $\begin{array}{l}\text { Informe con relato de } \\
\text { experiencia }\end{array}$ \\
\hline $\begin{array}{l}\text { Montero et al. } \\
\text { (2018) }\end{array}$ & Colombia & Foro en Moodle & $\begin{array}{l}\text { Tareas: según tema. Trabajo } \\
\text { colaborativo }\end{array}$ & Disciplinar & G & No & $\begin{array}{l}\text { Informe con relato de } \\
\text { experiencia }\end{array}$ \\
\hline $\begin{array}{l}\text { Rocu et al. } \\
\text { (2019) }\end{array}$ & España & WebQuest & Tarea: diseño colaborativo & Disciplinar & G & No & Informe de casos múltiples \\
\hline $\begin{array}{l}\text { Ubilla et al. } \\
\text { (2017) }\end{array}$ & Chile & GoogleDoc & $\begin{array}{l}\text { Tarea: creación colaborativa de } \\
\text { texto }\end{array}$ & Disciplinar & G & No & $\begin{array}{l}\text { Informe de estudio } \\
\text { experimental }\end{array}$ \\
\hline $\begin{array}{l}\text { Esquerra et al. } \\
\text { (2019) }\end{array}$ & España & Video & $\begin{array}{l}\text { Tarea: elaboración de video en } \\
\text { etapas }\end{array}$ & $\begin{array}{l}\text { Competencias digitales } \\
\text { para docentes }\end{array}$ & G & Si & $\begin{array}{l}\text { Informe con relato de } \\
\text { experiencia (Etapa 2) }\end{array}$ \\
\hline $\begin{array}{l}\text { Manso et al. } \\
\text { (2019) }\end{array}$ & España & Video & $\begin{array}{l}\text { Tarea: elaboración de video en } \\
\text { etapas }\end{array}$ & $\begin{array}{l}\text { Competencias digitales } \\
\text { para docentes }\end{array}$ & G & Sí & $\begin{array}{l}\text { Informe con relato de } \\
\text { experiencia (Etapa 1) }\end{array}$ \\
\hline $\begin{array}{l}\text { Sánchez et al. } \\
\text { (2019) }\end{array}$ & España & Video & Tarea: elaboración de video. & $\begin{array}{l}\text { Competencias digitales } \\
\text { para docentes }\end{array}$ & G & si & $\begin{array}{l}\text { Informe con relato de } \\
\text { experiencia }\end{array}$ \\
\hline $\begin{array}{l}\text { Basso et al. } \\
\text { (2018) }\end{array}$ & Chile & $\begin{array}{l}\text { Classroom, Drive y } \\
\text { Youtube }\end{array}$ & Modelo Flipped Classroom & $\begin{array}{l}\text { Competencias digitales } \\
\text { para docentes }\end{array}$ & ? & Si & $\begin{array}{l}\text { Presentación de modelo y } \\
\text { revisión de experiencias }\end{array}$ \\
\hline $\begin{array}{l}\text { Casado et al. } \\
\text { (2018) }\end{array}$ & España & Video & Video-lección y video-entrevista & $\begin{array}{l}\text { Competencias digitales } \\
\text { para docentes }\end{array}$ & $?$ & Si & Informe formato ensayo \\
\hline
\end{tabular}

Notas: 1 Grupal (G) o Individual (I). 2 Modelo FC = Flipped Classroom

Los artículos muestran tareas con consignas altamente pautadas con antelación enfatizando en algunos casos enfoques de tipo indagador. La mayor parte de los artículos, como puede apreciarse en el Cuadro 1, abordan trabajos grupales, destacando la posibilidad de construcciones colectivas con uso de TIC $(n=7)$. Se ilustra el uso de plataformas interactivas $(n=4)$ como Moodle y la elaboración de videos. Suelen abordarse temáticas disciplinares $(n=5)$ y competencias digitales para docentes $(n=6)$. Se destacan los relatos de experiencia, en algunos casos combinados con la presentación de un modelo, con múltiples casos o enfatizando la redacción ensayística $(n=9)$ por sobre los informes de investigación, ya sean de tipo cualitativo o experimental $(n=3)$.

Dada la importancia de la formación docente en las estrategias de enseñanza, particularmente en el campo de las TIC, haremos la descripción de los artículos tomando este eje. En primer lugar, describiremos las características de los escritos que se centran en la formación docente y luego analizaremos la importancia de las descripciones hechas en los propios textos en relación con este eje. En términos generales, intentaremos respetar el contenido de 
los escritos junto con las posiciones teórico-epistemológicas y metodológicas expresadas en cada escrito.

Manso et al. (2019) sostienen que el alumnado actual suele gestionar y comunicarse de modo audiovisual en su vida diaria, aunque este modo, desde el punto de vista académico, se encuentre escasamente integrado en los procesos de enseñanza y aprendizaje. Los autores señalan como motivo de esta situación la escasa formación del profesorado en este tipo de comunicación. Por esta razón, plantearon al profesorado en formación propuestas educativas que reunieran, por una parte, nuevas experiencias de aprendizaje de tipo no transmisivo y, por otra parte, nuevos modos de tratar los contenidos de manera más acorde a la sociedad de la información en la que vivimos. Propusieron la elaboración de un documental escolar sobre un tópico de ciencia a docentes en formación y analizaron cómo evolucionaron los contenidos propuestos en cada uno de los pasos seguidos en la actividad. Los autores sostienen que, a partir del cambio de formato de texto a video, el tratamiento de los contenidos cambió. La necesidad de redactar un guion forzó la incorporación de una trama argumental en la que integrar los contenidos escolares. Esta necesidad discursiva generó la búsqueda de situaciones reales y la consecuente ubicación de los contenidos escolares más cercanos al alumnado, facilitando la aparición de intereses propios, la vinculación entre el mundo cercano y el contexto académico y, todo ello, con su modo de expresarse. Por otra parte, la incorporación de imágenes obligó al grupo de docentes en formación a mostrar ordenadamente los hechos; tuvieron que considerar qué elementos visuales eran prioritarios, cuáles secundarios, cómo vincularlos, cómo indicar al espectador dónde mirar y cómo mirar, entre otros. En definitiva, la incorporación de lenguajes expresivos como el video posibilitó que estos procedimientos se constituyan en contenidos de aprendizaje en sí mismos.

La publicación de Ezquerra et al. (2019) puede ser leída en conjunto con la de Manso et al. (2019) dado que se trata de dos estudios del mismo caso. Este artículo también se centra en analizar las progresiones de futuros/as docentes en el tratamiento de contenidos cuando abordan la creación de audiovisuales educativos sobre cuestiones de ciencia. Desde la perspectiva de los autores, la creación de audiovisuales en el aula ha pasado a ser una herramienta de utilización masiva; sin embargo, una buena parte del profesorado actual no dispone de ejemplos vivenciales en su instrucción como estudiantes. La investigación presentada es un ejemplo vivencial destinado a analizar cómo incorporar los recursos audiovisuales en el aula. Trabajaron desde un enfoque indagador en el que cada docente en formación pudiera experimentar una labor investigadora que posteriormente podría llevar a cabo en un aula. El 
objetivo de este trabajo fue estudiar la evolución que experimentó el tratamiento de los contenidos cuando se produjo una modificación en el formato en el que son presentados; específicamente, cuando son transformados desde el texto al audiovisual. Hicieron un seguimiento de una serie de etapas que representaban procesos de aprendizaje por indagación. Los materiales producidos fueron estudiados mediante análisis cualitativo con categorías que permitieron identificar las progresiones en niveles de complejidad. Los resultados indican que la falta de referentes previos para esta tarea impulsó a los y las futuros/as docentes a indagar nuevos caminos. Desde el punto de vista de formadores de docentes, señalan Ezquerra et al. (2019), esto supondría que enfrentar a estudiantes a tareas alejadas de lo que les resulta familiar hace que abandonen sus roles estandarizados. Es decir, parece que el cambio de formato de texto a video en la generación de contenidos, en este caso, induciría a una evolución hacia un planteamiento menos estandarizado -es decir, más innovador- de los contenidos tratados.

Sánchez Vera et al. (2019) presentan las experiencias desarrolladas en varios cursos académicos entre el alumnado de magisterio de educación infantil y un conjunto de niños y niñas en torno a las estrategias vinculadas a contar cuentos (storytelling) en forma digital a través de videos, en el marco de un proyecto telecolaborativo. El proyecto se basó en la apertura del aula a otras aulas o centros educativos a través de redes, planteando actividades y tareas orientadas al enfoque metodológico de aprender haciendo y aprender interactuando. El storytelling, según se expresa en el texto, trasladado a entornos educativos recupera la idea del relato y la narrativa como un recurso a partir de herramientas multimedia digitales que permiten la combinación de gráficos, texto, grabaciones de video, audio y música para presentar información. Partiendo de la metodología del aprendizaje por tareas, el alumnado universitario diseñó un storytelling de manera grupal haciendo uso del video. Las historias eran planteadas a partir de una temática general propuesta por los niños y las niñas que, al mismo tiempo, elaboraban su historia digital. Todos los recursos fueron publicados en internet y difundidos a través de las redes sociales. La experiencia resultó enriquecedora ya que ambos grupos (universitario y de educación infantil) desarrollaron aprendizajes contextualizados y significativos, promoviendo la relación escuela-universidad. Diferentes estudiantes relataron que resultó motivador contactar con otros centros y comunicarse; la mayoría mencionó la complejidad de la tarea en cuanto al diseño del video a nivel técnico, pero tras la incertidumbre inicial, en general valoraron positivamente la experiencia. Los resultados muestran que el uso de storytelling integrado en experiencias educativas propicia el desarrollo 
de habilidades digitales (diseño de recursos audiovisuales), de capacidades lingüísticas y de la creatividad. La perspectiva de la creación de videos por parte de estudiantes no hace que el papel docente sea innecesario, más bien al contrario, ya que este ha de convertirse en guía en la facilitación del trabajo, proporcionando la formación que necesitan para desarrollar la historia desde el punto de vista técnico y pedagógico.

Casado (2018) sostiene que los mecanismos de aprendizaje necesitan ser replanteados dado que el aula física se ha convertido en un espacio limitado de generación del conocimiento. Tradicionalmente el recurso empleado para registrar la lección magistral ha sido la toma de apuntes por parte de cada estudiante. La mecánica habitual consiste en que alguien diserta y alguien registra esa disertación. Sin embargo, sostiene, el nuevo contexto socio-educativo y los constantes cambios estructurales a los que nos obliga la sociedad de la información demuestran que la toma de apuntes se está convirtiendo en un procedimiento mecanicista anacrónico, desfasado y, en términos de rentabilidad académica, un hábito improductivo. Por este motivo, expone las posibilidades que ofrece el material audiovisual en su implementación en educación superior. Desde la perspectiva del autor, la video-lección y la video-entrevista se están revelando como dos herramientas de enorme potencial que complementan las clases presenciales y dinamizan la transferencia de contenidos. De acuerdo al autor, como herramienta para transferir contenidos académicos, la amplia mayoría de estudiantes de nivel universitario se declara partidaria del material audiovisual por las múltiples ventajas y comodidades que supone recurrir a este tipo de formatos. A pesar de ello, la respuesta del profesorado universitario frente al video es dispar. Por esta razón, diseñó una encuesta, aplicada a nivel mundial, con el fin de poder sondear opiniones que puedan resultar concluyentes. La muestra estuvo constituida por historiadores/as y arqueólogos/as del ámbito científico-universitario de treinta países. Concluye que, en el ámbito de la transferencia académica, la herramienta que más prestaciones ofrece es la video-lección, que no solo facilita la retención de contenidos, sino que contribuye, en pleno auge de la cultura audiovisual, a la representación gráfica del conocimiento. El video, además, garantiza su permanencia y mejora la accesibilidad. Otra modalidad generadora de conocimiento es la video-entrevista, que permite interactuar con colegas mediante aportaciones académicas. Se trata de un nuevo reto que flexibiliza los cánones de enseñanza y aprendizaje desde la transversalidad académica, la creciente conectividad, el modelo cooperativo y las redes educativas virtuales. Asegura que se presenta como necesario dotar a las universidades de medios técnicos para la producción, grabación y edición que hagan viable y 
rentable el esfuerzo llevado a cabo por docentes. La solución, según Casado (2018), no pasa por exterminar la universidad presencial sino por maximizar el tiempo invertido por sus docentes y no entrar en un bucle de repetición de las mismas lecciones año tras año.

Desde el objetivo de brindar un soporte mediado por las TIC al modelo pedagógico existente, Basso et al. (2018) presentan una propuesta de modelo tecnológico para Flipped Classroom (FC) o aula invertida, denominado T-FliC. Este se basa en la utilización de recursos tecnológicos gratuitos cuya base son las aplicaciones de Google (Classroom, Drive y YouTube, entre otras). La elección se debe al uso masivo por parte de docentes y estudiantes de estas herramientas, lo que hace posible replicar este modelo en distintos contextos educativos. El modelo T-FliC considera una clase digital (aprendizaje fuera del aula) orientada de manera asincrónica por personal de tutoría virtual, un taller destinado al desarrollo de actividades dinámicas para el trabajo colaborativo (aprendizaje en el aula) guiado por personal de tutoría presencial y un proceso evaluativo mediado por herramientas tecnológicas dentro de las cuales se incluye un foro que permitiría tener el registro digital de la ruta de aprendizaje del estudiantado. Los autores realizan una revisión bibliográfica inicial acerca del rol de las TIC en los procesos educativos y de los fundamentos de la metodología Flipped Classroom (FC) incorporando antecedentes de experiencias de implementación de $\mathrm{FC}$ en educación superior y presentando el modelo T-FliC como una propuesta tecnológica para esta metodología.

El siguiente conjunto de artículos que describimos enfatizan la relación entre estrategias de enseñanza y TIC en estudios diversos. Si bien no se enfocan en la formación docente, los mismos constituyen estudios que aportan a la formación desde los resultados aportados tanto a nivel científico como en términos de inspiración para la labor docente.

Con el objetivo de aportar evidencia empírica a favor de la hipótesis de que el uso de la pizarra digital interactiva (PDI), como recurso didáctico en la enseñanza de la lectura, favorece la motivación y contribuye al desarrollo de competencias lectoras, Arancibia y Bustamante (2019) exploraron la incorporación de la misma durante un período de catorce semanas. La PDI es un sistema tecnológico que permite proyectar de modo interactivo contenidos digitales en un formato idóneo para la visualización grupal. Se utilizó un diseño cuasiexperimental con pre y postest y grupos intactos: uno participó como grupo de control activo (GC) y el otro como grupo experimental (GE). En el GC, la docente de aula implementó el programa de la asignatura haciendo uso de recursos didácticos que tradicionalmente se empleaban en el establecimiento. En el GE, la PDI se utilizó de manera sostenida en las clases. En contraste con el GC, el 
GE evidenció avances estadísticamente significativos entre pre y postest. Los resultados muestran que la integración didáctica de esta tecnología en situaciones interactivas de aprendizaje de la lectura contribuye al desarrollo de las actividades lectoras de los educandos, favoreciendo su interés y motivación.

Un estudio de caso múltiple realizado por Rocu et al. (2019) tuvo como propósito conocer el nivel de aprendizaje percibido y la satisfacción general con una experiencia educativa consistente en una propuesta expresivo-corporal implementada con WebQuest (WQ) en los niveles educativos de Primaria, Secundaria y Universidad. Una WQ, afirman los autores, es un recurso basado en las TIC que ha tenido cierta difusión en el ámbito educativo, especialmente en la universidad. Se trata de una herramienta que se encuentra disponible principalmente en internet, aunque también admite otros formatos, como documentos para presentación de diapositivas. La WQ se estructura en un conjunto de apartados concretos, en los que se propone al alumnado una tarea a resolver en grupos y se facilitan enlaces necesarios para transformar la información. Para Rocu et al. (2019), las bases teóricas que sustentan a las WQ se apoyan en teorías pedagógicas de larga data como, por ejemplo, el denominado Aprendizaje Basado en Proyectos (ABP). Los resultados obtenidos indican que la adquisición de aprendizajes a través de la WQ contempla la dimensión humana afectiva, estando presente en los argumentos del alumnado de los tres contextos educativos, puesto que reconocen como satisfactoria la adquisición de conocimientos desde el disfrute. Las conclusiones a las que arriban muestran que las competencias y objetivos didácticos diseñados en las propuestas educativas se han alcanzado adecuadamente, percibiendo haber aprendido especialmente conocimientos de tipo procedimental, tanto de la temática específica como tecnológicos a través de las WQ realizadas.

El artículo de Ubilla et al. (2017) reporta una investigación cuantitativa cuasiexperimental de clases intactas cuyo objetivo fue determinar el efecto de la escritura colaborativa en el desempeño de estudiantes de inglés al producir textos argumentativos. El grupo experimental (GE) trabajó con escritura colaborativa y el grupo control (GC) lo hizo con escritura individual. Ambos grupos utilizaron un procesador de textos comercial en línea durante doce sesiones en modalidad semipresencial. Los resultados de los postest mostraron un desempeño significativamente superior en los textos producidos por el GE. Se evidenció un efecto positivo del trabajo de escritura colaborativa de textos argumentativos en inglés mediado por la tecnología, ya que quienes escribían tenían la oportunidad de consultar su conocimiento explícito a sus pares y luego tomar decisiones más dialogadas en relación con el texto que se estaba construyendo. 
Montero y Kalman (2018) describen posibilidades y limitaciones de programas en línea desde el concepto de flexibilidad en términos del uso del tiempo. Analizaron los turnos conversacionales en un foro en línea de la plataforma Moodle de un grupo de cinco estudiantes que desarrollaban tareas académicas en un programa de formación a distancia en modalidad virtual. El análisis de los datos ilustró cómo la flexibilidad en el manejo del tiempo puede tomar diferentes matices. Se examinó la posibilidad que tenían de acceder a la plataforma en cualquier momento mientras lo hicieran dentro de los lapsos establecidos; la reconfiguración de los tiempos de las actividades de acuerdo con el trabajo que se venía desarrollando; y la exclusión de contribuciones individuales en el trabajo grupal a partir de conductas consideradas inapropiadas por el grupo. Más allá de las posibilidades que se encuentran en los discursos oficiales, se mostró que el carácter flexible es una construcción social entre integrantes del equipo, quienes articulan los lineamientos institucionales con sus expectativas sobre lo que es estudiar en línea, trabajar en equipo, producir una tarea y construir conocimiento. Los autores concluyen que la flexibilidad es una construcción compleja que involucra aspectos como el manejo del tiempo, la posibilidad de conectarse a los cursos desde cualquier lugar con acceso a internet y la definición de estrategias de estudio de acuerdo con los requerimientos de cada estudiante. Cabe señalar que este tipo de posibilidades es propiciado por el rol docente.

Jenaro et al. (2018) analizan la relación entre enseñanza, aprendizaje, TIC y rendimiento. Recolectaron todos los registros de las actividades realizadas por cada estudiante en la plataforma Moodle para luego analizar la asociación entre cantidad y tipo de accesos a la plataforma y rendimiento académico. Los resultados evidenciaron que el empleo de estrategias de enseñanza y aprendizaje activas se asoció con un mejor rendimiento. Demostraron la importancia de la implicación activa frente al mero acceso a la información disponible, es decir, no solo es importante tener acceso a documentos a través de las TIC, sino que el uso que se hace de ellas marca la diferencia en cuanto a los resultados obtenidos. En consecuencia, destacan que es clave fomentar la implicación activa en actividades en línea para lograr un aprendizaje más profundo.

Martínez y Chaín (2018) parten de la idea de que si bien las prácticas innovadoras en la red en enseñanza universitaria se consideran de gran interés, hay ámbitos académicos en los que es mucho más difícil ponerlas en marcha. Por este motivo, el objetivo de las autoras fue llevarlas a la práctica docente, usando sistemas ubicuos, en un posgrado de humanidades. Las tecnologías ubicuas aplicadas a la enseñanza tienen el fin de acercarse al estudiantado 
en cualquier sitio y a través de cualquier medio con el que pueda conectarse. Dentro de las ventajas de esta modalidad, las autoras señalan que podría ser una forma de aprender contenidos y herramientas digitales al mismo tiempo, dando la posibilidad de adquirir autonomía en la gestión de recursos virtuales. La tarea asignada consistió en generar y mantener durante todo el curso un blog y dos perfiles en redes sociales, elegidos por considerarse las plataformas de mayor uso y adaptabilidad. El propósito de la actividad consistió en averiguar si realmente la tarea de aprender a difundir lleva consigo una «nueva forma de aprender». El objetivo del estudio se centró en conocer si consideraban haber aprendido nuevas técnicas para gestionar herramientas 2.0 y si estaban utilizándolas a nivel profesional. Para averiguarlo, enviaron un cuestionario digital a sus estudiantes del posgrado. En los resultados se destacó que lo que más les gustó fue profundizar/investigar en temas de su interés y, sobre todo, interactuar con quienes leían y seguían los contenidos publicados. Lo que consideraron menos gratificante fue la inversión de tiempo requerida. Dentro de las ventajas de enseñar competencias digitales a estudiantes de humanidades, las autoras señalan el hecho de que no solo permiten brindarles más posibilidades dentro de esta sociedad de la información, sino que además se contribuye a generar experticia con suficientes conocimientos para que se conviertan en masa crítica sobre estas tecnologías que usan y conocen de cerca.

El artículo publicado por Aaron (2019) tiene como objetivo explicitar los procesos de enseñanza y aprendizaje a través de una didáctica caracterizada por la indagación, el acompañamiento permanente y el diálogo participativo con estudiantes repitentes del primer semestre de un programa de ingeniería. Según la autora, se deseaba que el grupo de estudiantes enfrentasen la metodología de aprendizaje combinado (blended learning), apoyada en la Plataforma Stanford Mobile Inquiry-based Learning Environment (SMILE) y lograr la generación de conceptos durante la construcción de preguntas derivadas de los contenidos. SMILE es una plataforma diseñada como entorno de aprendizaje basado en la indagación. Esta herramienta tecnológica unida a una didáctica activa pretende favorecer la acción pedagógica en un entorno virtual al que se accede desde internet. Desde el rol estudiante se construyen preguntas y también respuestas en la plataforma, lo que demanda conocimiento temático, coherencia en la escritura, sentido y comprensión. Aaron (2019) sostiene que la fusión entre lo presencial y lo virtual puede pensarse como estrategia de enseñanza. En este estudio, los resultados mostraron que diferentes estudiantes reconocían haber perdido la asignatura por no haber estudiado suficiente y no comprender los temas. Con el uso de la plataforma 
se generó un entorno de aprendizaje activo e interactivo ya que estos se vieron enfrentados no solo al reto de pensar un tema, sino también al de construir una pregunta y posibles respuestas, de las cuales una era la correcta, como así también al de responder las preguntas de sus compañeros/as.

\section{Discusión de los resultados y conclusiones}

La indagación realizada en este artículo se basó en entender que el modelo pedagógico y las modalidades físico-virtuales (exclusivamente presencial en persona, a distancia o bimodal) (Carranza y Caldera, 2018; Guarnieri, 2018; Ortiz, 2013; Villar, 2016) determinan la relación entre tecnologías y estrategias de enseñanza. Por esa razón, estudiamos ambos conceptos en uso en literatura científica especializada, analizando un total de 61 artículos.

En el primer conjunto de resultados se describieron características de los artículos en función del lugar (título, resumen o palabras clave) en el que aparecían las claves de búsqueda (TIC y estrategias) y otras variables construidas desde el análisis preliminar: mención de alguna TIC y/o estrategia de enseñanza en particular y si se trataba de un artículo con resultados empíricos o revisiones y reflexiones teóricas. En cuanto a la aparición de las claves de búsqueda, TIC se encontró, en orden de frecuencia descendente, en palabras clave, resumen y escasamente en título, mientras que estrategias estaba en resumen, título y palabras claves. La palabra estrategias aparece en estos lugares destacados de los artículos con una frecuencia menor que TIC, lo que puede deberse a que se mencionaban, en general, qué estrategias particulares se analizaban (por ejemplo: autoevaluación, creación de material audiovisual, aula invertida). Los relatos de experiencias educativas, análisis cualitativos, cuantitativos o experimentales de datos representaron la mayor parte de la muestra, siendo minoritarios los artículos que versaban sobre posicionamientos teóricos o sugerencias generales acerca de la temática abordada. Cabe destacar que esperábamos encontrar más escritos de este último tipo dado que la literatura sobre reflexiones generales acerca del uso de TIC en educación es abundante. A partir del análisis realizado, entendemos que esta particularidad de la muestra se debió a que se buscó simultáneamente ambas claves de búsqueda. Sería interesante realizar otros análisis exhaustivos de literatura para comprender mejor este fenómeno.

A partir de los primeros resultados descriptivos, construimos dos conjuntos de artículos para realizar un análisis cualitativo de sus características. El primer grupo fue constituido por los únicos cuatro artículos que mencionaban ambas claves de búsqueda en los lugares destacados (título, resumen o pa- 
labras clave). El segundo grupo fue compuesto por los escritos que versaban al mismo tiempo sobre una estrategia de enseñanza y una TIC en particular.

En cuanto a los resultados del primer grupo, artículos en los que aparecían ambas claves de búsqueda en lugares destacados, se referían a estudios sobre análisis institucionales del uso de TIC en educación (Carranza y Caldera, 2018; Marín et al., 2017) y a revisiones de literatura científica (Lizcano et al., 2019; Sánchez-Otero et al., 2019). Mencionaban que el uso de las TIC favorece el aprendizaje, hablándose de ellas en forma general y sin establecer demasiadas especificaciones en cuanto a aspectos tecnológicos o detalles de actividades que pueden ser pensadas a partir de aspectos tecnopedagógicos, como alcances y limitaciones de las tecnologías (Coll et al., 2007). Asimismo, también referían a las estrategias de enseñanza en términos generales, mencionando conceptos de aprendizaje asociados a teorías educativas reconocidas como el constructivismo (e.g. Lizcano et al., 2019). Destacaban, a su vez, la necesidad de reforzar las estrategias de enseñanza con integración de TIC.

Del segundo grupo de artículos, es decir, aquellos que hablaban simultáneamente de una TIC específica y una estrategia de enseñanza en particular, solían mostrar resultados propios del trabajo de investigación o docencia. Estos textos mostraban tareas con consignas altamente pautadas con antelación, enfatizando en algunos casos enfoques de tipo indagador, y una concepción de educación a distancia planificada (e.g. Sánchez-Vera et al., 2019; Manso et al., 2019). La mayor parte de estos escritos abordaban trabajos grupales, destacando la posibilidad de construcciones colectivas con uso de TIC (e.g. Ubilla et al., 2017); ilustraban el uso de plataformas interactivas y la elaboración de videos (e.g. Rocu et al., 2019; Casado, 2018), abordando temáticas disciplinares y competencias digitales para docentes (e.g. Ezquerra et al., 2019). Se destacaban los relatos de experiencia (e.g. Aaron, 2019), en algunas ocasiones, combinados con la presentación de un modelo o múltiples casos (e.g. Basso-Aránguiz et al., 2018). A su vez, enfatizaban la redacción ensayística por sobre los informes de investigación (e.g. Casado, 2018) ya sean de tipo cualitativo y/o experimental (e.g. Arancibia y Bustamante, 2019).

Como puede advertirse a partir de los conceptos abordados y resultados expuestos, el uso de TIC en relación con las estrategias de enseñanza direcciona las actividades docentes propiciando la construcción del aprendizaje.

Si bien en este trabajo no se abordó la enseñanza remota de emergencia dada en 2020 (Ramos, 2020; De Luca, 2020) como tema central, nos interesa reflexionar en relación con ese contexto debido al impacto que la virtualización forzosa de la educación ha tenido a nivel mundial. Los resultados muestran el énfasis de la educación a distancia como metódica, destacando 
planificación previa de materiales y actividades didácticas. Ejemplo de ello es el conjunto de artículos que exhiben resultados de trabajo de estudiantes con TIC y estrategias de enseñanza en particular (Arancibia y Bustamante, 2019; Sánchez-Vera et al., 2019; Manso et al., 2019). Asimismo, la educación a distancia pone énfasis en la interactividad y en la tendencia hacia la autonomía del estudiantado (Guarnieri, 2018). Esto último puede advertirse en el enfoque indagador (e.g. Ezquerra et al., 2019) o en estrategias como la del aula invertida (e.g. Basso-Aránguiz et al., 2018). Por tanto, al menos en el terreno de las consignas, la implementación de TIC en educación a distancia pareciera estructurar vertebralmente el trabajo docente. Estas características parecieran no haber estado presentes, al menos en los inicios, en la enseñanza remota de emergencia (De Luca, 2020; Ramos, 2020).

Tal como sostienen Manso et al. (2019), existe consenso en considerar que vivimos en una sociedad centrada en las TIC y con alta distribución de información. Sin embargo, afirman que esta misma sociedad reclama estrategias de enseñanza y aprendizaje innovadoras, aunque encuentra dificultades en cambiar los tradicionales estilos docentes centrados en la transmisión de conocimiento. La virtualización forzosa «arrojó» a la docencia al uso obligado de TIC; estudios futuros determinarán qué grado de impacto tuvo esta situación en las tradiciones de enseñanza a partir del ASPO (aislamiento social, preventivo y obligatorio).

En el contexto de pospandemia se requerirán aún más espacios de reflexión pedagógica ya reclamados por docentes respecto de las estrategias de enseñanza y su relación con las TIC (Borgobello et al., 2019). Si bien los resultados aquí presentados muestran la necesidad de reforzar las estrategias de enseñanza con integración de TIC por considerarlas beneficiosas en el trabajo pedagógico (Lizcano et al., 2019; Sánchez-Otero et al., 2019), estos cambios debieran darse en reflexiones colectivas y situadas.

De todos modos, entendemos como relevante enfocar las discusiones sobre uso de TIC y estrategias de enseñanza reflexionando desde los modelos pedagógicos (Ortiz, 2013) y las modalidades híbridas y a distancia. Sin embargo, a partir de la forzosa virtualización de la enseñanza (Copertari, 2020) el trabajo realizado «en exclusiva» en las aulas tradicionales de clase será probablemente una negación del grado de hibridación o mixtura de TIC y presencialidad dada la tendencia a los espacios físico-virtuales (Guarnieri, 2018).

En este sentido, si bien Anijovich y Mora (2010) reconocen la heterogeneidad de definiciones y conceptos asociados a las estrategias de enseñanza (como recursos, técnicas, procedimientos en relación con conceptos o/y aspectos institucionales), en esta conclusión se entienden en tanto decisiones, y 
abarcan al menos dos dimensiones: la dimensión de la reflexión y la dimensión de la acción. En esta misma línea, encontramos artículos que enfatizan una dimensión o la otra, aunque las mismas no sean excluyentes. En cuanto al énfasis en la dimensión de la reflexión por sobre la dimensión de la acción, podemos mencionar los artículos del primer grupo, especialmente aquellos que abordan revisiones de literatura científica (e.g. Lizcano et al., 2019). Por otro lado, y en un polo opuesto, es decir, si bien no quiere decir que construyan sus estudios sin considerar una dimensión reflexiva, encontramos textos con claro énfasis en la dimensión de la acción; entre ellos cabe mencionar algunos trabajos con escritura vinculada a informes con datos construidos en forma experimental (e.g. Arancibia y Bustamante, 2019).

Al poner en tensión los resultados relativos a los dos grupos de artículos analizados -aquellos que mencionaban en sitios destacados las claves de búsqueda y los que mencionaban estrategias y TIC en particular-, nos encontramos con que el primero enfatiza la dimensión de reflexión teórica e institucional mientras que el segundo enfatiza la dimensión de la acción directa en el aula. Queremos destacar que la mayor parte de los artículos analizados presentan cierto balance entre ambas dimensiones.

Adicionalmente, Anijovich y Mora (2010) mencionan que utilizan la denominación estrategias de enseñanza para destacar que no se trata de meras técnicas sino de decisiones enmarcadas en posicionamientos tanto teóricos como contextuales. Estos posicionamientos se ponen de manifiesto cabalmente en el primer grupo de textos analizados, estando dos vinculados a la descripción de cambios institucionales relacionados con la incorporación de TIC (Carranza y Caldera, 2018; Marín et al., 2017), y los otros dos, a estudios sobre literatura especializada en el tema (Lizcano et al., 2019; Sánchez-Otero et al., 2019).

El desarrollo y expansión de la web 2.0 permitió ampliar las potencialidades de la educación a distancia (García Aretio, 2007) dando lugar a la combinación dinámica de diversos lenguajes expresivos (Escandell et al., 2004) en formato de redes. Por tanto, las TIC acentuaron su posibilidad de comunicación además de la transmisión de información que caracterizaba a la histórica web 1.0. Estas potencialidades se vieron ampliamente plasmadas a partir de la forzosa virtualización en lo que De Luca (2020) mencionó como el adelanto de un futuro indeterminado, mostrando a su vez, las diferencias sociales de acceso a las tecnologías.

Retomando las ideas relacionadas con los posicionamientos teóricos y contextuales que cuestionan la idea de TIC en educación, entendemos la importancia de trabajar con conceptos vinculados específicamente con la inclusión social, la educación y el empoderamiento, como TIS, TEP y TAC (Cabero et al., 
2019; Guarnieri, 2018). Sin embargo, no encontramos en los textos referencia alguna a estos modos críticos de abordar la temática aquí trabajada.

Como señala Ortiz (2013), si bien suele ser escasamente definido, el modelo pedagógico reconoce un conjunto de representaciones ideales del mundo real educativo y se advierte en el trabajo concreto de planificación docente y acción en el aula. En algunos casos, como en el texto de Basso et al. (2018) sobre aula invertida, se profundiza a nivel teórico haciendo mención a conceptos vinculados al rol docente como guía, al trabajo grupal y al rol activo de estudiantes. En otros textos, como el de Arancibia y Bustamante (2019) sobre la implementación de una pizarra digital, se habla, por ejemplo, del modelo pedagógico tradicional haciendo referencia únicamente al uso de materiales no digitales.

En ese sentido, algunos textos parecieran quedarse en una descripción «no teórica». Desde nuestra perspectiva, esas representaciones idealizadas existen y se pueden abordar de modos más o menos conceptuales o teóricos. Los textos que tienen más profundidad conceptual refieren al constructivismo y a conceptos que pueden ser asociados al mismo como el aprendizaje colaborativo (e.g Lizcano et al., 2019). Entendemos a partir de los análisis realizados que la incorporación de TIC pareciera conllevar cierta idealización en relación con las prácticas pedagógicas, considerándolas, por ejemplo, motivadoras per se, siendo la dimensión crítica algo escasa.

En relación con esto último caben algunas reflexiones más. En primer lugar, los textos encontrados fueron escritos para destacar la labor docente con incorporación de TIC. Es posible que aquellas experiencias más negativas sean escasamente relatadas en escritos científicos. En segundo lugar, todos los textos fueron escritos y publicados con anterioridad a la pandemia de COVID-19 que generó, como se ha dicho, una forzosa virtualización de los sistemas educativos formales en prácticamente el mundo entero (De Luca, 2020).

Adicionalmente, tal como sostiene Díez (2012), las TIC pueden estar insertas en prácticas pedagógicas basadas en modelos interactivos constructivistas o en modelos transmisivos tradicionales sostenidos en la adquisición de conocimiento por parte del estudiantado. Sintéticamente, se puede innovar y ser constructivista sin utilizar TIC o, por el contrario, se puede ser tradicional utilizando TIC -probablemente el contexto de pandemia abunde en ejemplos de este último tipo-. Los resultados relativos al segundo grupo de artículos analizados muestran esta posibilidad de innovación y construcción de conocimiento con cierta claridad, destacando aspectos tecnopedagógicos (Coll et al., 2007) que vinculan de modos innovadores contenidos disciplinares y TIC en estrategias específicas de enseñanza basadas en contextos particulares. 
Para Coll et al. (2007), las TIC son instrumentos de tipo psicológicos en sentido vigotskiano. Es decir, no se trataría de tecnologías que se usan desde una exterioridad, sino que son interiorizadas. La incorporación de TIC abre posibilidades como así también muestra limitaciones, en un marco de normas, procedimientos y recomendaciones institucionales marcados desde diversos niveles del sistema educativo. A la vez, las actividades conjuntas generadas a partir de las estrategias de enseñanza con incorporación de TIC dan lugar a construcciones y negociaciones de significados basados en motivaciones y expectativas en escenarios que son únicos e irrepetibles. Por tanto, desde los análisis realizados y a partir de las propias experiencias, es posible afirmar que las maneras en que concebimos las TIC, incluso en sentidos más o menos conscientes y reflexivos, condicionan las estrategias pedagógicas mediadas por esas TIC.

Las tradiciones educativas muestran en el mundo previo a la pandemia de 2020 dos modalidades, la virtual y la presencial, y su combinación en esquemas bimodales (Villar, 2016). La pandemia, como se dijo, forzó la virtualización de las actividades pedagógicas presenciales en lo que se denominó enseñanza de emergencia (Ramos, 2020; De Luca, 2020), diferenciándose de ambas modalidades previas. Probablemente, además de las carencias relativas a la conectividad y los dispositivos para el trabajo pedagógico, gran parte de las limitaciones en las estrategias de enseñanza propuestas podrían haber sido más de índole psicológicas en sentido de Coll et al. (2007) que de disponibilidad de TIC.

A pesar de las limitaciones iniciales, las prácticas de diferentes docentes fueron interiorizando el trabajo con TIC, lo que auguraría un escenario educativo de pospandemia con mayor combinación de presencialidad y virtualidad. Tal como afirmaron Carranza y Caldera (2018), entendemos que esto implicará la combinación de estrategias de enseñanza provenientes de ambas tradiciones. Siguiendo a Duch y Núñez (2016), esa combinación dependerá de la centralidad de cada componente de la modalidad de la que se trate en consonancia con el modelo pedagógico asumido o subyacente. De acuerdo a nuestros resultados y a las ideas de Anijovich y Mora (2010), la relación entre selección de TIC y estrategias de enseñanza no siempre muestra exhaustivamente el modelo pedagógico desde el que se trabaja, centrándose más en la descripción de una TIC en particular que en la reflexión sobre los procesos de enseñanza y aprendizaje que tienen lugar (e.g. Rocu et al., 2019).

A modo de cierre, cabe destacar que, incluso, versiones superadoras de la escisión entre virtual (con TIC) y presencial (sin TIC) implican pensar no solo los modelos pedagógicos sino también que la incorporación de tecnologías 
en educación requiere de propuestas físico-virtuales y democratizadoras (Guarnieri, 2018).

Desde nuestra perspectiva, estudios posteriores deberían profundizar la relación entre modelos pedagógicos, objetivación y análisis de las estrategias de enseñanza con incorporación de TIC, especialmente a partir del impacto de la forzosa virtualización de la educación en tiempos de pandemia.

\section{Referencias bibliográficas}

AARÓN, M. A. (2019). Uso de la Plataforma Smile para la elaboración de conceptos en estudiantes en repitencia en un programa de ingeniería. Información Tecnológica, 30(2), 265-273. DOI: https://doi.org/10.4067/ S0718-07642019000200265

ANIJOVIC, R. y Mora, S. (2010). Estrategias de enseñanza. Otra mirada al quehacer en el aula. Buenos Aires: Aique.

ARANCIBIA-GUTIÉRREZ, B. y Bustamante-Molina, M. (2019). Aprendizaje lector con apoyo de la pizarra digital interactiva: estudio empírico. Magis, 12(24), 25-40. DOI: https://doi. org/10.11144/Javeriana.m12-24.alpd

ASINSTEN, J. C. (2013). Aulas expandidas: la potenciación de la educación presencial. Revista de la Universidad de la Salle, 60(1), 97-113. Disponible en: https://ciencia.lasalle.edu.co/cgi/viewcontent.cgi?article $=1354$ \&context $=$ ruls

BASSO-ARÁNGUIZ, M.; Bravo-Molina, M.; Castro-Riquelme, A. y Moraga-Contreras, C. (2018). Propuesta de Modelo Tecnológico para Flipped Classroom. Revista Electrónica Educare, 22(2), 1-17. DOI: https://doi. org/10.15359/ree.22-2.2

BorgobelLO, A.; Majul, S. y De Seta, D. (2019). Análisis cualitativo de una propuesta pedagógica para la incorporación de entor- nos virtuales a la enseñanza universitaria en Argentina a partir de tres ejes: experiencia, rol docente y tiempo. Hamut'ay, 6(3), 81-97. DOI: https://doi.org/http://dx.doi. org/10.21503/hamu.v6i3.1848

BORGOBELLO, A.; Raynaudo, G. y Peralta, N. (2014). Characterizing papers about blended learning: an unconsolidated field? Revista IRICE, 26, 37-66.

CABERO-ALMENARA, J.; Torres-Barzabal, L. y Hermosilla-Rodríguez, J. M. (2019). Las TIC y la creación de una ciudadanía crítica e-digital. Education in the Knowledge Society, 20(1), 1-10. DOI: https://doi.org/10.14201/ eks2019 20 a22

CABRERA-MEDINA, J. M.; Sánchez-Medina, I. I. y Rojas-Rojas, F. (2016). Uso de objetos virtuales de aprendizaje OVA como estrategia de enseñanza-aprendizaje inclusivo y complementario a los cursos teóricos-prácticos. Revista Educación en Ingeniería, 11(22), 4-12. DOI: https://educacioneningenieria.org/index.php/edi/article/ view/602

CARRANZA ALCÁNTAR, M. D. R. y Caldera Montes, J. F. (2018). Percepción de los estudiantes sobre el aprendizaje significativo y estrategias de enseñanza en el blended learning. REICE. Revista Iberoamerica- 
na Sobre Calidad, Eficacia y Cambio en Educación, 16(1), 73-88. DOI: https://doi. org/10.15366/reice2018.16.1.005

CASADO RIGALT, D. (2018). Un proyecto innovador en arqueología. El uso de material audiovisual como recurso didáctico en la enseñanza universitaria. Complutum, 29(2), 427-450. DOI: http://dx.doi.org/10.5209/ CMPL.62588

COLL, C.; Onrubia, J. y Mauri, T. (2007). Tecnología y prácticas pedagógicas: las TIC como instrumentos de mediación de la actividad conjunta de profesores y estudiantes. Anuario de Psicología, 38(3), 377-400.

CONCARI, S. (2014). Tecnologías emergentes ¿Cuáles usamos? Latin American Journal of Physics Education, 8 (3), 494-503. Disponible en: http://www.lajpe.org/sep14/13 LAJPE 899 Sonia Concari.pdf

COPERTARI, S. (2020). Virtualización de la educación en tiempos de pandemia. Revista Científica Educacao, 4(7), 891-895.

COPERTARI, S. y Sgreccia, N. (2011). Postgrados a distancia y virtualización en la Universidad Nacional del Rosario. Educ.Humanismo, 13(20), 14-32.

DELGADO FERNÁNDEZ, M. y Solano González, A. (2009). Estrategias didácticas creativas en entornos virtuales para el aprendizaje. Actualidades Investigativas en Educación, 9(2), 1-21.

DíEZ GUTIÉRREZ, E. J. (2012). Modelos socioconstructivistas y colaborativos en el uso de las TIC en la formación inicial del profesorado. Revista de Educación, 358(1), 175-196. Disponible en: http://hdl.handle. net/10612/2197

DUCH, V. A. y Núñez, S. I. (2016) Aulas presenciales y aulas virtuales: estrategias docen- tes compartidas, diferenciables y transferibles. En A. Villar (ed.), Bimodalidad (pp. 165-184). Articulación y Convergencia en la Educación Superior. Universidad Virtual de Quilmes.

ESCANDELL BERMÚDEZ, M. O.; Rodríguez Martín, A. y Cardona Hernández, G. (2004). Diversidad y Sociedad de la Información y el Conocimiento: las TIC como herramienta educativa. Anuario de Filosofía, Psicología y Sociología, 7, 95-106.

ESTÉFANO, R. (2018). Estrategias de Enseñanza y Aprendizaje para una Educación Inclusiva. En Z. Corredor, E. Ávila, M. Serradas, y A. Albarrán (coord.), Educación Inclusiva (pp. 351-377). Ediciones del Vicerrectorado Académico.

EZQUeRRA MARTíneZ, Á.; Burgos Jiménez, M. E., Manso Lorenzo, J. y Mafokozi, J. (2019). Análisis del tratamiento de contenidos en la creación de audiovisuales educativos (parte II): las progresiones detectadas. Revista Eureka Sobre Enseñanza y Divulgación de Las Ciencias, 16(2), 1-13. DOI: https://revistas.uca.es/index.php/eureka/article/view/4470/5270

GARCía ARETIO, L (2007). ¿Web 2.0 vs Web 1.0? Boletín Electrónico de Noticias de Educación a Distancia (BENED). Disponible en: http://e-spacio.uned.es/fez/view.php?pid=bibliuned:20094

GARCíA-PEÑALVO, F. J. y Ramírez-Montoyao, M. S. (2017). Aprendizaje, Innovación y Competitividad: La Sociedad del Aprendizaje. Revista de Educación a Distancia, 52(1), 1-6.

IBÁÑEZ-ETXEBERRIA, A.; Fontal Merillas, O. y Rivero Gracia, P. (2018). Educación patrimonial y TIC en España: marco normativo, 
variables estructurantes y programas referentes. Arbor, 194(788), 1-17. DOI: https:// doi.org/10.3989/arbor.2018.788n2008

JenARO Río, C.; Castaño Calle, R.; Martín Pastor, M. E. y Flores Robaina, N. (2018). Rendimiento académico en educación superior y su asociación con la participación activa en la plataforma Moodle. Estudios Sobre Educación, 34(1), 177-198. DOI: https://doi. org/10.15581/004.34.177-198

KOOHANG, A. (2004). Students' perceptions toward the use of the digital library in weekly web-based distance learning assignments portion of a hybrid programme. British Journal of Educational Technology, 35(5), 617-626.

LIZCANO-DALLOS, A. R.; Barbosa-Chacón, J. W. y Villamizar-Escobar, J. D. (2019). Aprendizaje colaborativo con apoyo en TIC: concepto, metodología y recursos. Magis, 12(24), 5-24. DOI: https://doi.org/10.11144/ Javeriana.m12-24.acat

LÓPEZ MOJICA, J. M.; Ramírez Cruz, J. C. y Aké Tec, L. P. (2018). La formación matemática de los futuros profesionistas de educación especial. En C. M. Salazar, C. S. Peña Vargas, y R. T. Medina Valencia (comp.), Estrategias de enseñanza y aprendizaje para la docencia universitaria (pp. 53-66). Colima: Universidad de Colima.

LUCUMI USEDA, P. y González Castañeda, M. (2015). El ambiente digital en la comunicación, la actitud y las estrategias pedagógicas utilizadas por docentes. Tecné Episteme y Didaxis: TED, (37), 109-129. DOI: https:// doi.org/10.17227/01213814.37ted109.129

MANSO-LORENZO, J.; Ezquerra, Á.; Burgos-Jiménez, M. E. y Mafokozi, J. (2019). Análisis del tratamiento de contenidos en la crea- ción de audiovisuales educativos. Revista Eureka Sobre Enseñanza y Divulgación de Las Ciencias, 16(1), 1-16. DOI: https:// Castelsarrasin/index.php/eureka/article/ view/4193

MARÍN, F. V.; Inciarte, A. de J.; Hernández, H. G. y Pitre, R. C. (2017). Estrategias de las instituciones de educación superior para la integración de las tecnología de la información y la Comunicación y de la innovación en los procesos de enseñanza. Un estudio en el distrito de Barranquilla, Colombia. Formación Universitaria, 10(6), 29-38. DOI: https:// doi.org/10.4067/S0718-50062017000600004

MARTín, R. D. y Tourón, J. (2017). El enfoque flipped learning en estudios de magisterio: percepción de los alumnos. Revista Iberoamericana de Educación a Distancia, 20(2), 187-211. DOI: https://doi.org/10.5944/ ried.20.2.17704

MaRTínez USARRALDE, M. J.; López Martín, R. y Pérez Carbonell, A. (2018). E-innovación en Educación Superior. Claves para la institucionalización en las universidades. PixelBit, Revista de Medios y Educación, 52(1), 183-197. DOI: https://doi.org/10.12795/ pixelbit.2018.i52.13

MARTÍNEZ-PALMERA, O.; Combita-Niño, H. y De-La-Hoz-Franco, E. (2018). Mediación de los objetos virtuales de aprendizaje en el desarrollo de competencias matemáticas en estudiantes de ingeniería. Formación Universitaria, 11(6), 63-74. DOI: http://dx. doi.org/10.4067/S0718-50062018000600063

MARTíNEZ-SOLís, L. y Chaín-Navarro, C. (2018). Humanidades digitales para el aprendizaje y difusión del Patrimonio Naval. Revista de Educación a Distancia, 57(9), 1-19. DOI: https://doi.org/10.6018/red/57/9 
MATESANZ DEL BARRIO, M. y Ferreira Martins, V. (2017). Tecnologías para la enseñanza de lenguas extranjeras: Una contribución para lograr los retos educativos de Brasil. Calidoscopio, 15(1), 141-154. DOI: https:// doi.org/10.4013/cld.2017.151.11

MIRANDA TORRES, L. A. (2015). Estrategias pedagógicas mediadas con las TIC-TAC, como facilitadoras del aprendizaje significativo y autónomo. Palobra, 18(1), 214-241. Disponible en: http://hdl.handle.net/11227/7778

MIRANDA-CALDERÓN, L. A.; Angulo-Hernández, L. M. y Román-López, G. (2018). El programa Perfiles, dinámicas y desafíos de la educación costarricense: Una propuesta para la innovación pedagógica y la producción de materiales y recursos didácticos tecnológicos en la Universidad Nacional, Costa Rica. Revista Eletrónica Educare, 22(1), 1-24. DOI: https://doi.org/10.15359/ree.22-1.10

MONTERO, R. y Kalman, J. (2018). Construcción de la flexibilidad en una universidad colombiana pública a distancia con modalidad virtual. Revista de Educación a Distancia, (58). DOI: https://doi.org/10.6018/ $\underline{\mathrm{red} / 58 / 8}$

NOSSA SÁNCHEZ, J. (2007). Efectos diferenciales de dos estrategias didácticas sobre el aprendizaje en estudiantes universitarios. Suma psicológica, 14(2), 289-312.

ORTIZ OCAÑA, A. (2013). Modelos pedagógicos y teorías del aprendizaje. ¿Cómo elaborar el modelo pedagógico de la institución educativa? Ediciones de la U.

PÉREZ ESCOBAR, C. (2020, 10 de abril). El gran test de las clases 'online'. La Tercera. Disponible en: https://www.latercera.com/tendencias/noticia/el-gran-test-de-las-clasesonline/JOJOMO7S2BAB3FNRJYPPHGUZ3I/
RAMÓN, J. y Vilchez, J. (2019). Tecnología Étnico-Digital: Recursos Didácticos Convergentes en el Desarrollo de Competencias Matemáticas en los Estudiantes de Zona Rural. Información Tecnológica, 30(3), 257268. DOI: http://dx.doi.org/10.4067/S0718$\underline{07642019000300257}$

RAMOS TORRES, D. (2020). El Coronateaching como síndrome. Disponible en: https:// www.iesalc.unesco.org/2020/07/02/coronateaching-sindrome-o-nueva-oportunidad-para-la-reflexion-i-ii/

Río, C. J.; Castaño Calle, R. C.; Martín Pastor, M. E. y Robaina, N. F. (2018). Rendimiento académico en educación superior y su asociación con la participación activa en la plataforma Moodle. Estudios Sobre Educación, 34, 177-198. DOI: https://doi. org/10.15581/004.34.177-198

RIVERO CÁRDENAS, I.; Gómez Zermeño, M. y Abrego Tijerina, F. (2013). Tecnologías educativas y estrategias didácticas: criterios de selección. Educación y Tecnología, 3(1), 190-206.

ROCU GÓMEZ, P.; Blández Ángel, J. y Sierra Zamorano, M. Á. (2019). Construyendo aprendizajes en Expresión Corporal a través de WebQuest: Un estudio de caso múltiple Patricia. Revista Complutense de Educación, 30(4), 1013-1029. DOI: https://doi. org/10.5209/rced.59905

SÁNCHEZ OTERO, M.; García-Guiliany, J.; Steffens-Sanabria, E. y Hernández-Palma, H. (2019). Estrategias Pedagógicas en Procesos de Enseñanza y Aprendizaje en la Educación Superior incluyendo Tecnologías de la Información y las Comunicaciones. Información Tecnológica, 30(3), 277-286. DOI: https://doi. org/10.4067/s0718-07642019000300277 
SÁNCHEZ VERA, M. del M.; Solano, I. M. y Recio, S. (2019). El storytelling digital a través de vídeos en el contexto de la Educación Infantil. Pixel-Bit: Revista de Medios y Educación, 54(1), 165-184. Disponible en: https://recyt.fecyt.es/index.php/pixel/article/ view/65102/42104

TURIZO MARTíNEZ, L. (2011). Las estrategias pedagógicas, una alternativa para solucionar dificultades académicas. Revista Pensamiento Americano, 2(6), 45-50.

UBILLA ROSALES, L.; Gómez Álvarez, L. y Sáez Carrillo, K. (2017). Escritura colaborativa de textos argumentativos en inglés usando Google Drive. Estudios Pedagógicos, 43(1), 331-348. DOI: https://doi.org/10.4067/ S0718-07052017000100019

VALVERDE, M. T. (2018). Escritura académica con Tecnologías de la Información y la Comunicación en Educación Superior. Re- vista de Educación a Distancia, 58(1), 1-21. DOI: https://doi.org/10.6018/red/58/14 VALVERDE-CRESPO, D.; Pro-Bueno, A. de, y González-Sánchez, J. (2018). La competencia informacional-digital en la enseñanza y aprendizaje de las ciencias en la educación secundaria obligatoria actual: una revisión teórica. Revista Eureka Sobre Enseñanza y Divulgación de las Ciencias, 15(2), 1-15. DOI: https://revistas.uca.es/index.php/ eureka/article/view/4193

VENTURA, A. C. (2013). La investigación sobre los estilos de enseñanza. Aportes para mejorar la didáctica de ciencias. Revista Digital de Investigación en Docencia Universitaria, 1(7), 9-18. DOI: https://doi.org/10.19083/ ridu.7.183

VILLAR, A. (ed.) (2016). Bimodalidad. Articulación y Convergencia en la Educación Superior. Universidad Virtual de Quilmes. 